This item was submitted to Loughborough's Research Repository by the author.

Items in Figshare are protected by copyright, with all rights reserved, unless otherwise indicated.

\title{
Uniqueness below a cut-off frequency for the two-dimensional linear water-
} wave problem

PLEASE CITE THE PUBLISHED VERSION

LICENCE

CC BY-NC-ND 4.0

\section{REPOSITORY RECORD}

Mclver, M.. 2019. "Uniqueness Below a Cut-off Frequency for the Two-dimensional Linear Water-wave Problem”. figshare. https://hdl.handle.net/2134/805. 
This item was submitted to Loughborough's Institutional Repository (https://dspace.lboro.ac.uk/) by the author and is made available under the following Creative Commons Licence conditions.

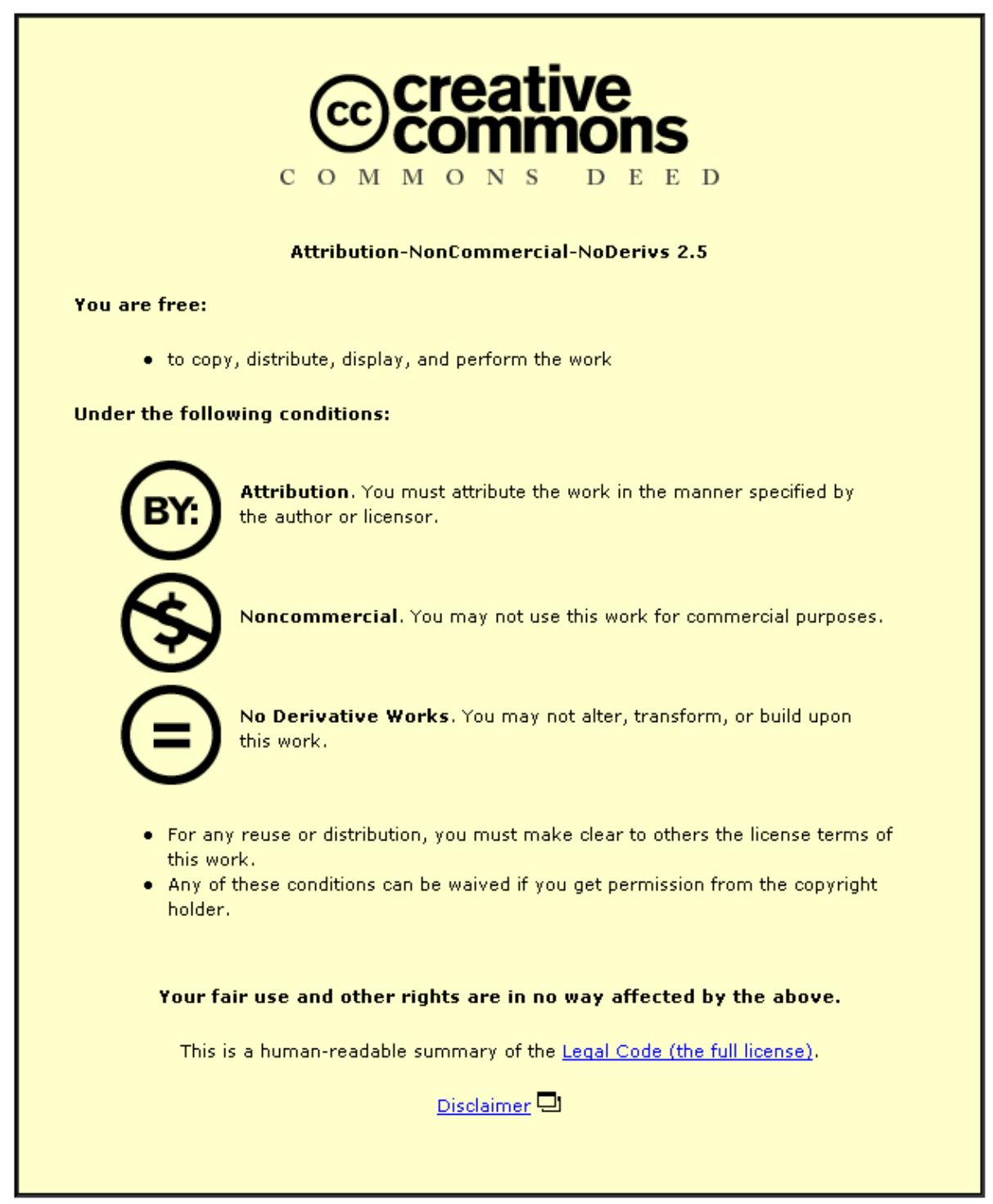

For the full text of this licence, please go to: http://creativecommons.org/licenses/by-nc-nd/2.5/ 


\title{
Loughborough $\checkmark$ University
}

\section{Uniqueness below a cut-off frequency for the two-dimensional linear water-wave problem}

\author{
B Y M. MCIVER \\ Department of Mathematical Sciences, Loughborough University, Loughborough, \\ Leics., LE11 3TU, UK
}

The two-dimensional velocity potential associated with the scattering of linear waves in water of finite but non-uniform depth is shown to be unique for $K h_{\max } \leq 1$. Here $h_{\max }$ is the maximum depth of the fluid and $K=\omega^{2} / g$, where $\omega$ is the angular frequency and $g$ is the acceleration due to gravity. Uniqueness is established by proving that the homogeneous boundary value problem for the velocity potential $\phi$ has only the trivial solution $\phi=0$ when $K h_{\max } \leq 1$.

It is first shown that there is a nodal line in the fluid on which $\phi=0$ and that this line has one end on the mean free surface and asymptotes to a horizontal line as $x \rightarrow \infty$. The line forms the lower boundary of a fluid region contained between itself and the free surface. The potential in this region is shown to be identically equal to zero for $K d_{\max }<1$, where $d_{\max }$ is the maximum depth of the layer, by bounding the potential energy in the region by a fraction of the kinetic energy. By analytic continuation the potential is zero in the whole fluid for this range of frequencies and as $d_{\max }<h_{\max }$, uniqueness is established for $K h_{\max } \leq 1$. The critical value of $K d_{\max }=1$ corresponds to a cut-off frequency below which waves cannot propagate in a uniform layer of depth $d_{\max }$ with $\phi=0$ on the lower boundary.

The analysis is extended to prove uniqueness for the same range of frequencies when any number of non-bulbous, surface-piercing bodies are in the fluid or a single surface-piercing or submerged body with arbitrary shape.

\section{Introduction}

The discovery that trapped modes can exist in the two-dimensional, linear water-wave problem by McIver (1996) has increased interest in the problem of determining under what circumstances the scattering potential associated with a system of bodies is unique. (If trapped modes exist at a particular frequency then the corresponding boundary value problem for forced motion at that frequency does not have a unique solution.) A review of the systems of bodies and ranges of frequencies for which uniqueness of potential has been established is given by McIver (1996). By constructing a potential which has all the properties of a trapped mode she was able to show that a trapped mode exists in the presence of a pair of special, two-dimensional bodies which are precisely spaced in the surface of a deep fluid. Her work was subsequently extended by Kuznetsov \& Porter (1997) who showed that trapped modes could also exist in the presence of multiple cylinders in finite depth and by McIver \& McIver (1997) who showed that 
three-dimensional trapped modes could be supported by a floating torus. More recently Evans \& Porter (1998) have shown that structures which are formed from combinations of two surface-piercing bodies and one submerged body can also trap two-dimensional waves.

Trapped modes are known to exist in many other types of boundary value problems and a classical example is the Stokes edge wave (Stokes 1846) which is trapped above a sloping beach and propagates along the shoreline. Ursell (1952) showed that this edge wave is the first of a series of trapped modes, the number of which depends on the angle of the beach. Ursell (1951) also showed that waves may be trapped above a submerged, horizontal cylinder and propagate along the cylinder. More recently Evans, Levitin \& Vassiliev (1994) proved that trapped modes exist in the presence of bodies which are symmetrically placed in waterwave channels or guides. Unlike the modes found by McIver (1996), these other types of trapped modes occur at frequencies which are less than a 'cut-off' value, below which waves cannot propagate to infinity. In the terminology of spectral theory, the trapped modes occur at frequencies which correspond to eigenvalues below the bottom of the continuous spectrum for the problem and they may often be shown to exist with the use of a variational principle. In the two-dimensional water-wave problem there is no cut-off as waves may propagate to infinity at any frequency. This is one reason why it is difficult to prove the existence or otherwise of trapped modes in this problem.

There is not, in fact, a cut-off frequency below which no waves can propagate to infinity in a waveguide but Evans, Levitin \& Vassiliev (1994) were able to introduce an artificial cut-off frequency by restricting attention to motion which is antisymmetric about the centreline of the channel. The restriction to antisymmetric motion is equivalent to the requirement that the centreline of the channel should be a nodal line on which $\phi=0$. (A discussion of the properties of nodal lines in bounded domains is given in Courant \& Hilbert 1953.) In the next section the existence of a nodal line in the two-dimensional water-wave problem is established and in $\S 3$ the existence of this line is shown to introduce an artificial cut-off frequency into the problem. Uniqueness is established for frequencies below the cut-off in fluid layers which have variable depth and the analysis is extended in $\S 4$ to prove uniqueness for the same range of frequencies when bodies are introduced into the fluid.

\section{Existence of a nodal line}

Localised small oscillations are sought in a two-dimensional fluid layer $\Omega$ which has variable depth, as illustrated in figure 1 . The fluid is assumed to be inviscid and incompressible and the motion irrotational and oscillatory with angular frequency $\omega$. Thus the flow is described by a velocity potential $\operatorname{Re}\left[\phi(x, y) \mathrm{e}^{-i \omega t}\right]$ where $\phi \in C^{2}(\Omega) \cap C(\bar{\Omega})$ and satisfies

$$
\nabla^{2} \phi=0
$$

and the linearised free surface condition

$$
K \phi+\frac{\partial \phi}{\partial y}=0 \quad \text { on } \quad y=0,
$$

Proc. R. Soc. Lond. A (1998) 


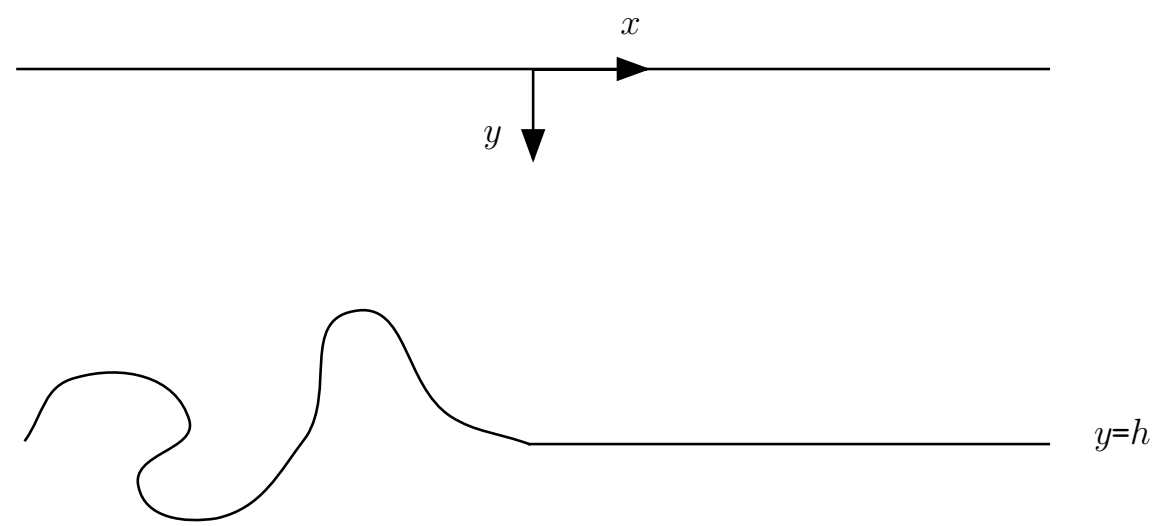

Figure 1. Definition sketch.

where $K=\omega^{2} / g$ and $g$ is the acceleration due to gravity. Axes are chosen so that the origin is in the mean free surface and the $y$-axis points vertically downwards. The layer is assumed to have constant depth $h$ in the region $x \geq 0$ but may vary in a piecewise smooth fashion in $|x|<0$. It is not necessary that the depth of water should tend to a constant value as $x \rightarrow-\infty$, but it must be finite and non-zero everywhere. There is no flow through any rigid surface and so

$$
\frac{\partial \phi}{\partial n}=0 \quad \text { on the sea-bed, }
$$

where $\partial / \partial n$ denotes the derivative in the outward normal direction. Trapped modes are localised oscillations which have finite energy and so if $\phi$ corresponds to a trapped mode then

$$
\int_{\Omega}|\nabla \phi|^{2} \mathrm{~d} V+K \int_{-\infty}^{\infty}|\phi(x, 0)|^{2} \mathrm{~d} x<\infty .
$$

In particular this means that

$$
\phi \rightarrow 0 \quad \text { as } \quad|x| \rightarrow \infty
$$

Uniqueness is established if the only solution to $(2.1)-(2.5)$ is the trivial solution $\phi=0$. Conversely, if there is a non-zero solution to (2.1)-(2.5) it is a trapped mode and any forced problem for this geometry does not have a unique solution at this frequency.

Without loss of generality $\phi$ is assumed to be real because if it were complex then both the real and imaginary parts would separately satisfy the governing equations and boundary conditions. The harmonic function

$$
\psi=\sin k(x-b) \cosh k(y-h),
$$

where $k h$ is the real, positive root of the dispersion relation $K h=k h \tanh k h$, satisfies the same boundary conditions on the free surface and sea-bed as $\phi$ in the region $x \geq b$ where $b \geq 0$. In addition $\psi$ is bounded as $x \rightarrow \infty$ and so an application of Green's third identity

$$
\int_{D}\left[\phi \nabla^{2} \psi-\psi \nabla^{2} \phi\right] \mathrm{d} V=\int_{\partial D}\left[\phi \frac{\partial \psi}{\partial n}-\psi \frac{\partial \phi}{\partial n}\right] \mathrm{d} S
$$

Proc. R. Soc. Lond. A (1998) 
to $\phi$ and $\psi$ in the region $x \geq b$ gives

$$
\int_{0}^{h} \phi(b, y) \cosh k(y-h) \mathrm{d} y=0 .
$$

The function $\cosh k(y-h)$ is strictly positive and as $\phi(b, y)$ is a continuous function of $y$,

$$
\phi\left(b, y_{0}\right)=0
$$

for some $y_{0}(b)$ such that $0<y_{0}(b)<h$. A value of $y_{0}$ may be found for every $b \geq 0$ and so by continuity, there is at least one nodal line on which $\phi=0$ in the region $x \geq 0$. Moreover in $x \geq 0, \phi$ is represented by the eigenfunction expansion

$$
\phi=\sum_{n=1}^{\infty} a_{n} \cos k_{n}(y-h) \mathrm{e}^{-k_{n} x}
$$

where $\left\{k_{n} h\right\}$ is the monotonically increasing sequence of positive roots of the dispersion relation

$$
K h=-k_{n} h \tan k_{n} h
$$

and $\left\{a_{n}\right\}$ is a sequence of constants. If $\phi$ is not identically equal to zero in the fluid then for $x / h \gg 1$ it is dominated by the first non-zero term in the series in (2.10) and so

$$
\phi=a_{j} \cos k_{j}(y-h) \mathrm{e}^{-k_{j} x}+O\left(\mathrm{e}^{-k_{j+1} x}\right) \quad \text { as } \quad x \rightarrow \infty,
$$

for some $j$. For large, fixed values of $x, \phi(x, y)$ varies like $\cos k_{j}(y-h)$ with the possible exception of exponentially small regions near the zeros of this function. However, as $\phi$ changes from positive to negative values on either side of these regions, there is a nodal line on which $\phi=0$ which asymptotes to the line $y=d$ as $x \rightarrow \infty$, where $k_{j} d$ is the smallest, positive root of the equation

$$
\cos \left(k_{j} h-k_{j} d\right)=0 \text {. }
$$

(A value $d<h$ exists as, from the dispersion relation (2.11), $k_{j} h \geq k_{1} h>\pi / 2$.)

As $\nabla \phi$ is perpendicular to the lines $\phi=$ const, the local tangent to the nodal line $\phi=0$ is parallel to the vector $\left(-\phi_{y}, \phi_{x}\right)$ and so the nodal line is smooth with the possible exception of points at which $\phi_{x}=\phi_{y}=0$. A similar argument to that used by McIver (1996) shows that the nodal line cannot terminate in the interior of the fluid but must end on the free surface or the sea-bed or both of the ends of the line must go to infinity. If the line terminates on the sea-bed or if both of its ends go to infinity then a region $D_{\infty}$ in the fluid is formed which is open to infinity and contained within lines on which either $\phi=0$ or $\partial \phi / \partial n=0$. An application of the divergence theorem in this region yields

$$
\int_{D_{\infty}}(\nabla \phi)^{2} \mathrm{~d} V=\int_{\partial D_{\infty}} \phi \frac{\partial \phi}{\partial n} \mathrm{~d} S=0
$$

which means that $\phi$ is a constant throughout $D_{\infty}$ and as $\phi=0$ on the nodal line, this constant is equal to zero. By analytic continuation $\phi$ is zero throughout the whole fluid. Thus for $\phi$ to represent a trapped mode, the nodal line which asymptotes to the line $y=d$ as $x \rightarrow \infty$ must end on the free surface. Although the precise position of the line is unknown, it defines the lower boundary of a subregion of the fluid which is contained between it and the free surface. The 
maximum depth of the region, $d_{\max }$ is less than the maximum depth of the fluid $h_{\max }$ and it will be shown in the next section that trapped modes do not exist in this region for $K d_{\max }<1$ and by analytic continuation, do not exist in the whole fluid for $K h_{\max } \leq 1$.

\section{Uniqueness of solution for $K h_{\max } \leq 1$}

The velocity potential for waves which propagate in a fluid layer of uniform depth $d$, which satisfies the condition $\phi=0$ on the lower boundary is given by

$$
\phi=\sinh p(y-d) e^{ \pm i p x},
$$

where, to satisfy the free surface condition $(2.2), p d$ is a root of the dispersion relation

$$
K d=p d \operatorname{coth} p d .
$$

As $z \operatorname{coth} z \geq 1$ for real $z$, there are no real values of $p d$ which satisfy (3.2) if $K d<1$. Thus, there is a cut-off frequency below which waves cannot propagate in a uniform layer if $\phi=0$ on the lower boundary.

The region $D_{0}$ is defined to be the region contained between the nodal line and the free surface and the coordinate axes are redefined so that the origin is at the intersection of the nodal line and the free surface. A similar method to that used by John (1950) is followed and $\partial \phi / \partial y$ is integrated down a vertical line from a point $(s, 0)$ on the free surface of this new region, to give

$$
\phi(s, 0)=-\int_{0}^{d(s)} \frac{\partial \phi}{\partial y}(s, y) \mathrm{d} y,
$$

where $d(s)$ is the smallest value of $y$ such that the point $(s, d(s))$ lies on the nodal line. (If there is only one such value then $y=d(s)$ is the equation of the nodal line.) Both sides of (3.3) are squared and the Cauchy-Schwarz inequality gives

$$
\phi^{2}(s, 0) \leq\left[\int_{0}^{d(s)} 1^{2} \mathrm{~d} y\right]\left[\int_{0}^{d(s)}\left(\frac{\partial \phi}{\partial y}\right)^{2} \mathrm{~d} y\right] \leq d_{\max } \int_{0}^{d(s)}\left(\frac{\partial \phi}{\partial y}\right)^{2} \mathrm{~d} y,
$$

where $d_{\max }$ is the maximum depth of the nodal line. Substitution of the free surface condition (2.2) and the boundary condition $\phi=0$ on the nodal line into the first equation in (2.14) (which is valid for a general fluid region) gives

$$
\begin{aligned}
\int_{D_{0}}(\nabla \phi)^{2} \mathrm{dV} & =K \int_{0}^{\infty} \phi^{2}(x, 0) \mathrm{d} x \\
& \leq K d_{\max } \int_{0}^{\infty} \int_{0}^{d(x)}\left(\frac{\partial \phi}{\partial y}\right)^{2} \mathrm{~d} y \mathrm{~d} x \leq K d_{\max } \int_{D_{0}}(\nabla \phi)^{2} \mathrm{~d} V
\end{aligned}
$$

from (3.4). If $K d_{\max }<1$ then the inequality in (3.5) is only satisfied if $(\nabla \phi)^{2}$ is identically equal to zero which means that $\phi$ is a constant and this constant must be zero from the nodal line condition. So there are no trapped modes in the subregion if $K d_{\max }<1$. As $d_{\max }<h_{\max }$, the maximum depth of the fluid, there are no trapped modes in the subregion and by analytic continuation the whole fluid, if $K h_{\max } \leq 1$. 


\section{Variable topography with bodies}

The proof of uniqueness for $K h_{\max } \leq 1$ extends to the situation in which there are a finite number of non-bulbous, surface-piercing, piecewise smooth bodies in a fluid layer of variable depth. In this case the axes are chosen so that all bodies and bed variations lie in the region $|x| \leq 0$. The same analysis as used in $\S 2$ shows that a nodal line exists which asymptotes to the line $y=d$ as $x \rightarrow \infty$. However, when there are bodies in the fluid, it is possible that the nodal line may end on one of the bodies instead of the free surface, or it may end on the free surface between two of the bodies. In both of these scenarios, however, a subregion of the fluid is formed which has the nodal line and possibly a portion of one of the bodies, as its lower boundary. If the bodies are non-bulbous then vertical lines may be extended from every point on the free surface in the subregion to the nodal line and the analysis of the previous section applies.

The proof of uniqueness for $K h_{\max } \leq 1$ also extends to the case where there is a single, piecewise smooth, submerged or surface-piercing body of any shape, in a fluid layer which only has depth variations in a finite region. For this configuration, there is also a nodal line which asymptotes to the line $y=$ const as $x \rightarrow-\infty$ and if both nodal lines end on the body then an application of (2.14) in the fluid region below the nodal lines shows that the potential is identically equal to zero everywhere. Thus, at least one of the nodal lines must end on the free surface and this defines a subregion of the fluid in which the argument of the previous section may be applied.

\section{Discussion}

Uniqueness has been established in the two-dimensional water-wave problem for variable fluid layers, at frequencies which satisfy $K h_{\max } \leq 1$. This extends the results of Kreisel (1949), Vainberg \& Maz'ja (1973), Fitz-Gerald (1976) and FitzGerald \& Grimshaw (1979), all of whom proved uniqueness with either geometric restrictions on the sea-bed profile or for restricted ranges of frequencies. In addition, the proof of uniqueness for the same range of frequencies has been shown to apply to the case where there are a finite number of non-bulbous, surface-piercing bodies in the fluid.

It is clear from the work of Kuznetsov \& Porter (1997) that the condition that the bodies should be non-bulbous is crucial. Kuznetsov \& Porter (1997) placed two finite depth wave sources in the free surface, at a separation of one half of a wavelength and determined the streamline pattern of the flow. By interpreting parts of the streamlines as body boundaries they were able to construct pairs of bulbous bodies in a uniform layer for which trapped modes exist. Although the numerical results which they presented were for $K h>1$, where $h$ is the depth of the layer, the analysis is applicable for arbitrary $K h$ and so trapped modes may exist in the presence of bulbous bodies for $K h \leq 1$.

The analysis presented here does not apply to the pair of surface-piercing, bulbous bodies which were shown to support trapped modes by McIver (1996), as the fluid depth is not finite in that case. However, the numerical evidence is that there is a nodal line which asymptotes to the line $K y=1$ as $x \rightarrow \infty$, which terminates on the right hand body and which satisfies $K y<1$ everywhere. One physical reason for the existence of this trapped mode, in the subregion which is 
surrounded by a portion of the body, the nodal line and the free surface, is that the body forms a beach which can support waves but that the waves are unable to propagate to infinity as they cannot be supported above the nodal line.

There are numerical indications that trapped modes exist for pairs of nonbulbous bodies in fluid of infinite depth. These bodies are constructed from portions of streamlines, using the method of McIver (1996), but the potential contains wave-free singularities in addition to the wave sources. In this case the nodal line must satisfy $K y>1$ over at least part of its range. Work is currently in progress to determine the shape of the nodal lines for various configurations of bodies which support trapped modes.

Although a cut-off frequency has been introduced into the two-dimensional water-wave problem, it has not yet been possible to prove the existence of a trapped mode below the cut-off, with the use of a variational principle, for even a limited class of bodies. This is because, even if the existence of a trapped mode in a given subregion of the fluid is established, in general this trapped mode does not correspond to a trapped mode in the full fluid domain. This is in contrast to the situation in a wave guide, where Evans, Levitin \& Vassiliev (1994) were able to exploit the symmetry of the guide and body configuration to show that a trapped mode in the half-guide which satisfies $\phi=0$ on the centreline is also a trapped mode in the full guide. Further work is in progress to determine whether or not the existence of nodal lines can be used to prove the existence of trapped modes in the two-dimensional water-wave problem.

\section{References}

Courant, R. \& Hilbert, D. 1953. Methods of Mathematical Physics I, John Wiley \& Sons.

Evans, D. V., Levitin, M. \& Vassiliev, D. 1994. Existence theorems for trapped modes. Journal of Fluid Mechanics 261, 21-31.

Evans, D. V. \& Porter, R. 1998. An example of non-uniqueness in the two-dimensional linear water wave problem involving a submerged body. Submitted to Proceedings of the Royal Society London A.

Fitz-Gerald, G. F. 1976. The reflection of plane gravity waves travelling in water of variable depth. Philosophical Transactions of the Royal Society of London, A 284, 49-89.

Fitz-Gerald, G. F. \& Grimshaw, R. H. J. 1979. A note on the uniqueness of small-amplitude water waves travelling in a region of varying depth. Mathematical Proceedings of the Cambridge Philosophical Society 86, 511-519.

John, F. 1950. On the motion of floating bodies, II, Communications in Pure and Applied Mathematics 3, 45-101.

Kreisel, G. 1949. Surface waves. Quarterly of Applied Mathematics 7, 21-44.

Kuznetsov, N. G. \& Porter, R. 1997. Uniqueness and trapped modes in the two-dimensional water-wave problem. Submitted to European Journal of Applied Mathematics.

McIver, M. 1996. An example of non-uniqueness in the two-dimensional linear water wave problem. Journal of Fluid Mechanics 315, 257-266.

McIver, P. \& McIver, M. 1997. Trapped modes in an axisymmetric water-wave problem Quarterly Journal of Mechanics and Applied Mathematics 50, 165-178.

Stokes, G. G. 1846. Report on recent researches in hydrodynamics Brit. Ass. Rep., 1-20.

Ursell, F. 1951. Trapping modes in the theory of surface waves Proceedings of the Cambridge Philosophical Society 47, 347-358.

UrSell, F. 1952. Edge waves on a sloping beach Proceedings of the Royal Society London A 214, 79-97.

Proc. R. Soc. Lond. A (1998) 
VAinBerG, B.R. \& MAZ'JA,V.G. 1973. On the problem of the steady state oscillations of a fluid layer of variable depth. Transactions of the Moscow Mathematical Society 28, 56-73. 